\title{
MIMO OFDM channel estimation based on RLS algorithm: the time- versus frequency- domain implementations
}

\begin{abstract}
In this paper, exponentially-weighted recursive least squared (EW-RLS) channel estimation for multiple-input multiple-output (MIMO) orthogonal frequency division multiplexing (OFDM) systems is investigated. Channel estimation in time-domain (TD) as well as frequency-domain (FD) is investigated through exploiting preambles and pilot symbols inserted in TD and FD, respectively. The channel is assumed to be slowly time-varying frequency-selective, constant during one OFDM symbol but changing from symbol to symbol. Simulation results show that the TD EW-RLS estimator has better estimation performance in terms of the mean-squares error (MSE) and bit-error rate (BER), compared to FD EW-RLS estimation approach. The computational complexity is significantly reduced by recursively updating the channel estimates and by applying the matrix inversion lemma.
\end{abstract}

Keyword: Channel estimation; Time-domain (TD); Frequency-domain (FD); MIMO; OFDM; Exponentially-weighted recursive least squared (EW-RLS) 